ones during the post-operative period; the writer has seen serious trouble from this cause.

Acute appendicitis with peritonitis. The straightforward case of appendicitis diagnosed early does not require any intravenous fluids. Once severe peritoneal irritation has occurred, there is an increased danger of paralytic ileus developing. In treating such cases intestinal suction is used. The volume of fluid aspirated must be replaced by the intravenous route, giving the solution of sodium and potassium chloride described earlier.

In summary, fluid balance can be maintained in intestinal obstruction in children without many laboratory investigations, so long as the measurements of the volumes of fluid removed by suction, lost at operation, and given intravenously are made accurately.

\section{BIBLIOGRAPHY}

DODD, $K$, and RAPOPORT, S. (1950), 'Mitchell-Nelson Textbook of Pediatrics,' 5th edition, p. 201; W. B. Saunders Company.

GROSS, R. E., and FERGUSON, C. C. (1952), Surg. Gynaec. \& Obstet., 95, 631 .

KEMPTON, J. J. (195 I), Brit. med. F., i, 1140.

LEE, C. M. (1951), Ann. Surg., 134, 1066.

\title{
FLUID BALANCE IN INTESTINAL OBSTRUCTION
}

\author{
By L. P. LE Quesne, F.R.C.S. \\ Department of Surgical Studies, Middlesex Hospital, London, W.I
}

The last 20 years have seen a great improvement in the results of treatment of patients with acute intestinal obstruction. In the main this improvement is due to an increasing understanding of the importance of the two basic pathological effects of obstruction, namely, intestinal distension, and the loss from the body of large quantities of fluid and electrolytes. It is now well recognized that in the treatment of intestinal obstruction the relief of this distension and the replacement of fluid losses play as great a part in the successful outcome of the case as the release of the obstruction itself. In many cases the management of the patient's water and electrolyte equilibrium will pose very complex problems, and in all cases an exact analysis of the exchanges taking place will require intricate biochemical calculations, but these considerations must not be allowed to obscure the fact that the great majority of these cases can be successfully handled by attention to a few simple concepts.

\section{The Nature of the Fluid Losses}

The crux of the problem of the maintenance of fluid and electrolyte equilibrium in patients with intestinal obstruction consists in the assessment and replacement of the abnormal losses which occur from the alimentary tract. These abnormal losses consist of the secretions into the intestinal canal, which accumulate proximal to the obstruction and are to a varying extent vomited up, or
Table I

\begin{tabular}{|c|c|c|c|}
\hline \multicolumn{3}{|c|}{ Source } & Volume \\
\hline $\begin{array}{l}\text { Saliva } \\
\text { Gastric secretion } \\
\text { Bile . . } \\
\text { Pancreatic juice } \\
\text { Secretion of intesti }\end{array}$ & $\begin{array}{cc}\cdots & \cdots \\
\cdots & \cdots \\
\cdots & \cdots \\
\ddot{a} & \cdots \\
\text { nal mucosa }\end{array}$ & $\begin{array}{l}\cdots \\
\cdots \\
\cdots \\
\cdots\end{array}$ & $\begin{array}{r}1500 \mathrm{ccs} . \\
2500 \mathrm{ccs} . \\
500 \mathrm{ccs} . \\
700 \mathrm{ccs} . \\
3000 \mathrm{ccs} .\end{array}$ \\
\hline Total .. & . & .. & 8200 ccs. \\
\hline Jormal Plasma Vol & . & . & 3500 ccs. \\
\hline
\end{tabular}

The normal volume of digestive juices secreted per 24 hours, by an adult. (From Gamble, 1950.)

removed by intestinal suction. Certain salient facts about these secretions must be borne in mind. First, the total volume of fluid secreted into the alimentary tract each 24 hours amounts to over 8 litres (Table $\mathrm{I}$ ). When it is remembered that the normal circulating plasma volume is only 3 litres, it is apparent that losses from the intestine can readily and rapidly lead to a dangerously large fluid deficit. Secondly, such losses consist not of water but of a solution of electrolytes which, though varying in composition in each secretion, is in all of them essentially isotonic with the plasma electrolytes. Fig. I shows the composition of these secretions in their pure form, but as lost from the body in intestinal obstruction they are, of course, diluted to a variable extent by ingested water, and Table 2 


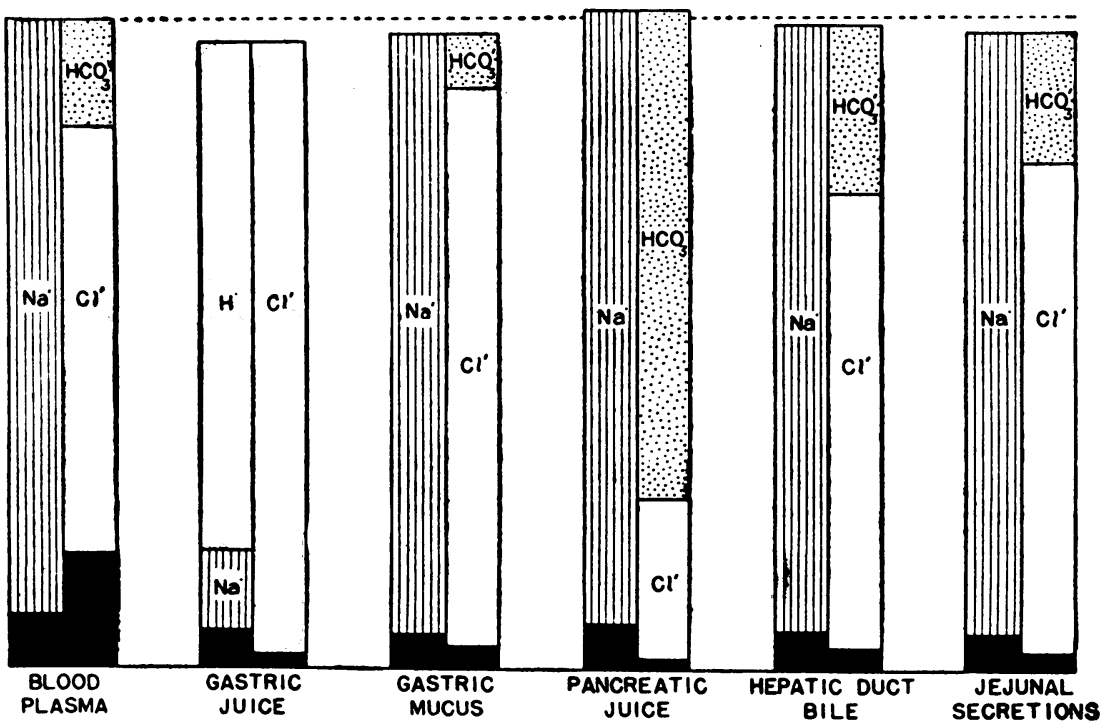

Fig. I.-Composition of the intestinal secretions in their pure form (from Gamble, 1950).

TABLE 2

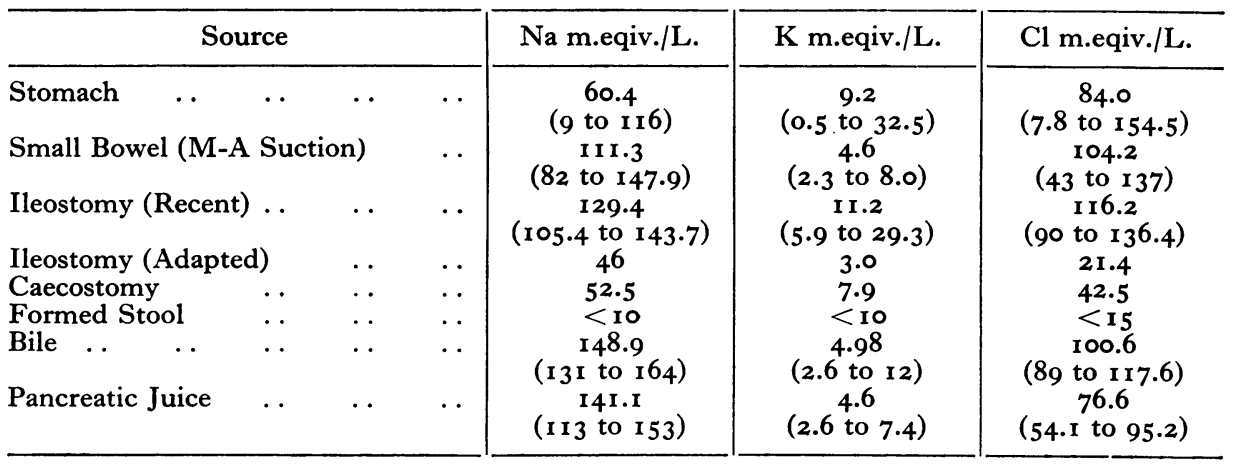

(From Lockwood and Randall, 1949)

Table showing the Electrolyte Losses in Fluids from Various Parts OF THE Alimentary Tract

The upper figure is an average, whilst those in brackets show the range of observed values.

shows the composition of the losses from the various portions of the alimentary tract as they occur in clinical practice: even under these circumstances the fluids, particularly a mixture of gastric and intestinal juices, are nearly isotonic and so must be replaced, not by water or dextrose solutions, but by an appropriate electrolyte solution. Thirdly, it is important to remember that these intestinal fluids contain potassium in a concentration up to three times that in the plasma, so that their loss from the body can cause a relatively large potassium deficit which may well require replacement.
The Effect of Losses from the Alimentary Tract

As a result of the loss from the body of these intestinal fluids and also because of the interference with the normal intake caused by the obstruction, there will develop a combined deficit of water and salt (sodium). The clinical picture will therefore contain the features of both these deficits, but in order to recognize the significant features of this mixed state it is necessary to consider separately the fundamentally differing effects of water depletion and salt depletion. Initially water depletion causes hypertonicity in the extracellular space, 

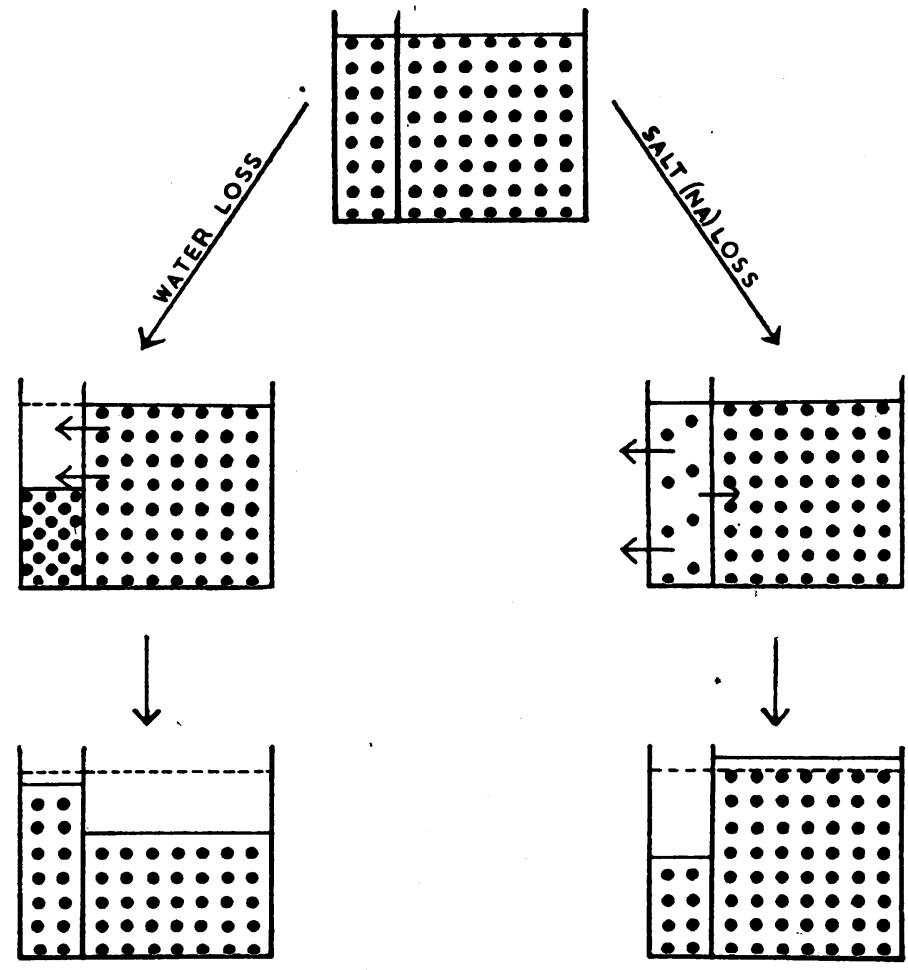

INTRACELLULAR DEHYDAATION

\section{EXTRACELLULAR} DEHYDRATION

Fig. 2.-Diagrammatic representation of the difference between water and salt depletion. The body water is represented as a tank, divided into the smaller extracellular space and larger intracellular space: the dots represent the ions in solution. Water loss initially causes extracellular hypertonicity; to compensate for this there is a shift of water out of the cells, resulting in intracellular dehydration. Salt loss initially causes extracellular hypotonicity; this is compensated for partly by a shift of water into the cells, but mainly by a renal excretion of water, leading to extracellular dehydration. (Reproduced from the author's 'Fluid Balance in Surgical Practice,' by kind permission of Messrs. LloydLuke (Medical Books), Ltd., London.)

leading to a shift of water out of the intracellular space and so resulting in intracellular dehydration: by contrast, salt (sodium) depletion causes initially an extracellular hypotonicity, leading to an excretion of water by the kidneys in an effort to restore extracellular tonicity, and so resulting in extracellular dehydration (see Fig.2). This extracellular dehydration does not result in equal contraction of the two portions of the extracellular space, namely, the interstitial space and the plasma space, as at first the osmotic force of the plasma proteins maintains the plasma volume at the expense of the interstitial space; but as the condition progresses this mechanism eventually breaks down, resulting in a contracting plasma volume and finally peripheral circulatory failure.

These differences in the type of dehydration caused are reflected in the different clinical and biochemical effects of the two losses (see Fig. 3).
The predominant clinical feature of water deple- $\frac{3}{3}$. tion is increasing thirst, and there are no striking 욱 physical signs: as would be expected the urine is sparse, with a raised specific gravity (above r.oro) and a high salt content; as the brunt of the loss falls on the intracellular space there are no 은 significant changes in the blood chemistry until $D$ late in the condition, when there will be a steady rise in the haemoglobin, plasma protein and plasma N electrolyte concentrations, the only exception to this being the blood urea concentration which $\tilde{O}$ will rise steadily from the start owing to the $\underset{\omega}{ }$ inadequate urine output. In salt depletion thirst $\mathscr{\sigma}$ is noticeably absent, but as the condition pro- 0 gresses the patient becomes increasingly lethargic $\stackrel{D}{\Phi}$ and nauseated, with postural giddiness and $\stackrel{\odot}{+}$ eventually extreme weakness. In the early stages $T$ the important physical findings are dryness of the skin and mouth with diminishing skin elasticity, 

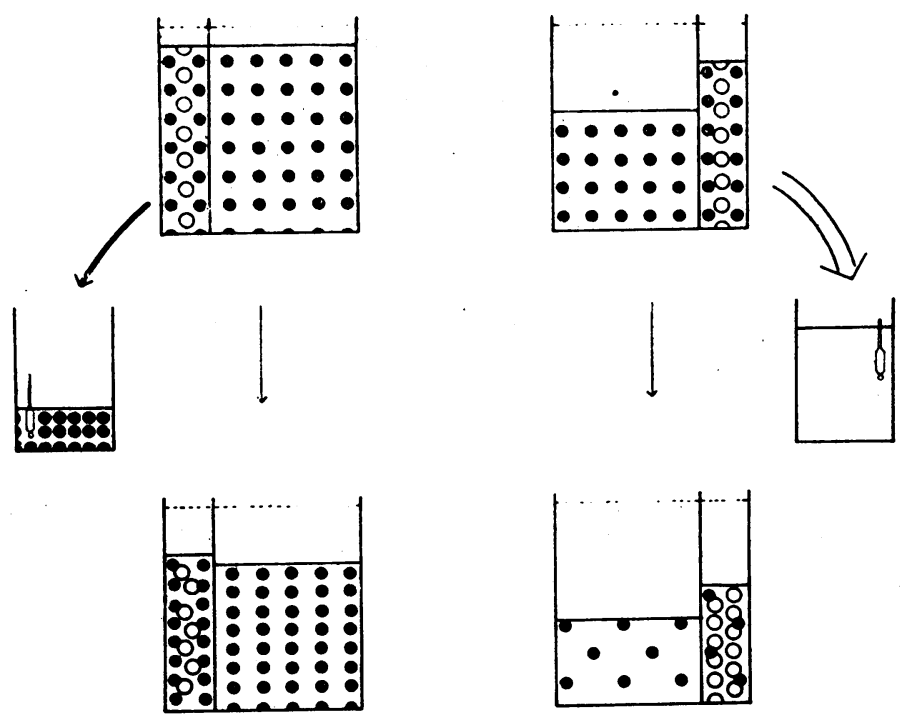

Fig. 3. - The tank represents the extracellular space, divided into its two component portions, the larger interstitial space and smaller intravascular (or plasma) space. The dots represent the ions in solution and the circles the plasma proteins. The containers to either side represent the urine output. In water depletion there is a scanty urine output of high specific gravity and electrolyte (chloride) content; late in the condition there is a rise in both the plasma protein and plasma electrolyte figures. In salt depletion the urine output is copious of a low gravity, but the most significant finding is a lowered or completely absent electrolyte (chloride) content. In the early stages of salt depletion the brunt of the loss falls on the interstitial space, but later the plasma volume falls markedly: the plasma protein concentration rises steadily throughout the condition, but only late is there a significant fall in the plasma electrolyte figures. (Reproduced by kind permission of the editors of the Proceedings of the Royal Society of Medicine.)

and later as the blood volume begins to fall there will be postural hypotension progressing to the complete picture of peripheral circulatory failure. Provided there is no associated water depletion the urine volume will be within normal limits until late on, of a low specific gravity, but with a diminished or absent chloride content (normal, $>$ 3om.eq/1.). The most significant blood findings are a steadily rising haemoglobin and plasma protein concentration, and it is only late in the condition that the plasma electrolyte concentrations fall; it cannot be too strongly emphasized that a large deficit of sodium and/or chloride can readily exist with normal plasma levels of these ions. As in water depletion, the blood urea will show a steady rise, particularly late in the condition when there is an interference with renal function.

Two other effects of the loss of intestinal fluids require note. As already mentioned, in some of the intestinal secretions the concentrations of sodium and chloride are not equal, so that losses of these fluids can lead to disproportionate deficits of either sodium or chloride, resulting in an acidbase disturbance. In most cases of intestinal obstruction the fluid lost is a mixture of the various secretions containing essentially equivalent amounts of sodium and chloride (Table 2), so that the problem does not arise, and even in the few cases in which an acid-base disturbance does complicate the picture it rarely requires specific treatment. A further complication is provided by the deficit of potassium which may arise as a result of severe losses from the alimentary tract. The main effect of this deficit is to lead to marked depression of muscular activity resulting in the muscular hypotonus, the paralytic ileus, the lowered plasma $\mathrm{K}$ level, and the E.C.G. changes which together form the important diagnostic features of the condition. It is now apparent that in many cases of obstruction the recognition and replacement of such a potassium deficiency is of great importance, but nevertheless in all cases the problem of overriding importance is the correct replacement of the salt and water deficit and this must always receive prior attention.

\section{The Aims of Treatment}

In nearly every case of intestinal obstruction it is necessary to give the patient his fluids intravenously or by some other parenteral route. The aim of this treatment is twofold, to provide the patient with the normal daily requirements of water and electrolytes, and in addition to replace quantitatively the abnormal fluid loss from the 
intestines. The administration of a reasonable basic daily intake is the first requirement in the treatment of patients with fluid and electrolyte disturbances. This basic intake is designed to replace the normal loss of water and electrolytes from the skin and lungs, and from the kidneys as urine. Such requirements are met by an intake of 3 litres of water containing 4-5 g. of $\mathrm{NaCl}$ per 24 hours. This can be given quite simply intravenously by giving each 24 hours $2 \frac{1}{2}$ litres of 5 per cent. dextrose and 0.5 litre of normal saline, and provided there are no abnormal losses this allows of a urine output of about $1,500-1,700 \mathrm{ml}$. per 24 hours, of a specific gravity below 1.010, and with a chloride concentration of about $40 \mathrm{~m} . \mathrm{eq} / \mathrm{l}$. Under normal circumstances there is much to be said for adding some potassium to the basic intake, but this should only be done when there is a good urine output. In most cases of intestinal obstruction, at least in the early stages, the urine output is sparse so that such an addition is not permissible, and accordingly it is wiser to omit potassium from the basic intake in these cases, and to give it when indicated as part of the quantitative replacement of abnormal losses. It cannot be stressed too strongly that the regular administration of a sensible, daily intake is the essential first step in the management of fluid disturbances arising in intestinal obstruction. Additional fluids will be required to replace the abnormal loss from the intestines, but such replacement is useless unless superimposed upon a regular, rational provision for the losses by normal routes.

\section{The Replacement of Abnormal Losses}

In the replacement of the abnormal losses from the alimentary tract two distinctly different problems arise. When a patient with intestinal obstruction comes under treatment he is likely to have an established deficit of water and electrolytes, due mainly to losses into and from the alimentary tract and partly to an inadequate or absent basic intake since the start of the illness. Once treatment has been started there should be no further deficit owing to an inadequate basic intake, but the abnormal losses may well continue for several days. These 'observed losses' which occur whilst the patient is under treatment can to a great extent be measured, and their replacement is comparatively simple. But the 'pre-existing losses 'cannot be measured, so that their assessment and replacement creates a much more difficult problem. In clinical practice the replacement of both these losses must go on simultaneously, but they involve such different methods that they must be considered separately.

The first step in the replacement of pre-existing losses is to arrive at an estimate of their size and type. There is no one single test which will give this information, which can only be obtained by a careful clinical and biochemical examination of $\frac{\varrho}{c}$ the patient. The object of this examination is to detect the stigmata of water and salt depletion $\stackrel{\vec{F}}{\rightarrow}$ described previously, and to dissect out from the $\bar{C}$ whole picture the evidence indicative of one or both of these deficits. In this process care must be taken not to get overwhelmed in a mass of detailed and possibly conflicting information and it is important to concentrate upon the essential signs. In the clinical examination the signs of salt depletion should be carefully looked for, and it must not be forgotten that in itself this deficit, when marked, can cause peripheral circulatory failure. In the urine the finding of a specific gravity above 1.015, provided there is no sugar present, is practically diagnostic of water depletion, and a chloride concentration below $20 \mathrm{~m} . \mathrm{eq} / \mathrm{l}$. is equally strong evidence of salt depletion (but see below for effect of operation on these findings). The most important observations on the blood are the estimation of the haemoglobin and/or plasma protein concentrations, which will be raised in salt depletion, and of the blood urea which is a very sensitive index of depletion as it is raised by both salt and water deficits. In assessing the signifio cance of the haemoglobin figure the possibility of a co-existent anaemia must be remembered. IT general, plasma electrolyte concentrations nee $\overrightarrow{\mathrm{d}}^{+}$ only be estimated in complicated cases, and it must be clearly recognized that large deficits of both salt and water can exist with normal plasma electrolyte concentrations. On the basis of the history and the findings just reviewed it will be possible to decide on the type of deficit present or predominating, and some estimate of the size can usually be made. In reaching this last figure, which can only be approximate, it is useful to remember that when the signs of depletion are obvious the deficit amounts to about 5 per cent. of the body-weight, so that under these circumstances an average-sized man (70 kg.: I I stone) will be short of at least 4 litres of fluid, of which at least half will be an extracellular deficit requiring replacement by saline.

Once an estimate of the size of the pre-existing deficit has been arrived at the requisite amount of 5 per cent. dextrose and normal saline should be added to the basic intake over the ensuing 24-48 hours. Every case must be considered as a therapeutic trial, and it is only by the repetition of the initial examinations and tests that the adequacy of the treatment can be judged and further additions made as indicated. The process of replenishment may be considered complete when there are no longer any clinical signs of 
depletion, when the urine volume is normal, with a chloride concentration in excess of $40 \mathrm{~m} . e q / \mathrm{l}$. and a specific gravity below 1.010, and when the blood chemistry is steady within normal levels.

The replacement of observed losses is a much simpler problem. All abnormal losses from the alimentary tract must be accurately measured and then replaced volume for volume with normal saline. In view of the fact that the intestinal losses are somewhat hypotonic this method of replacement is likely to give somewhat too much salt, so that if in any 24-hour period the abnormal losses exceed 2 litres, the fifth $\frac{1}{2}$-litre bottle of replacement fluid should consist of glucose not saline. In practice it is most convenient to make these adjustmènts to cover observed losses every 12 hours, adding to the intake for the ensuing I2-hour period an amount of saline equal to the abnormal losses during the previous 12 hours. Thus every 12 hours the intake for the next period is calculated by adding to the basic intake (a) any fluid required to replace a pre-existing deficit, (b) any fluid required to replace observed losses, and the adequacy of the intake can be accurately checked by observations on the urine volume, etc., as explained in the last paragraph.

\section{Potassium Depletion}

Patients with intestinal obstruction are liable to develop potassium depletion for two reasons: (a) the losses from the alimentary tract contain potassium in a concentration up to three times that in the plasma (Table 2), and (b) there is a constant loss of potassium from the kidneys, which do not conserve this ion very zealously, even when the intake is zero; further, if the patient has to undergo an operation the loss in the urine will be greatly increased by the potassium diuresis which always occurs in the first 48 hours post-operatively. Experience in the past has shown that the majority of patients with intestinal obstruction will recover without any attention being paid to this potassium deficit, but in the minority it certainly can and does give rise to serious trouble. Accordingly, it is wise to be constantly aware of this possibility and whenever possible to administer potassium before a serious deficit has developed.

Potassium depletion should always be suspected in any patient who has abnormal losses over a period of days during which the intake of this ion is negligible or zero. Clinically the diagnosis will be suggested by muscular weakness and hypotonia, and the development of a chronic ileus, and it can be confirmed either by the presence of characteristic E.C.G. changes (for a description of these changes see Lewis, Somerville and Le Quesne, 1954), or by a lowering of the serum K level. The normal serum $K$ concentration is
3.9-4.5 m.eq/l., and findings below $3.5 \mathrm{~m} . \mathrm{eq} / \mathrm{l}$. are highly suggestive of a deficit. Whenever possible potassium salts should be given by mouth, and they should not be given intravenously in the presence of a marked deficit of water or salt, or to any patient who has an inadequate urine output (less than $1,000 \mathrm{ml}$. per 24 hours) or poor renal function. Accordingly, in the treatment of preexisting losses the first requirement is the replacement of the salt-and-water deficit, and it is only when this has been done that the potassium deficit should be sought for and treated if required. In the replacement of observed losses it is only necessary to add potassium to the replacement fluid if the losses continue for over 48 hours, or are in excess of 2 litres in any given 24-hour period, though again this addition must only be made if the urine output is adequate. Apart from those already mentioned, certain other precautions should be taken when giving potassium salts intravenously. The total daily dosage should not exceed 80 m.eq., the concentration in which they are given should not exceed $40 \mathrm{~m} . e q / \mathrm{l}$., and the rate of administration should not be in excess of $20 \mathrm{~m} . e q / \mathrm{hr}$. As regards the actual solutions used, considerable experience has shown the following two simple formulae to be most useful: (I) $\mathrm{NaCl} 2.25 \mathrm{~g}$. per litre, $\mathrm{KCl} 3.0 \mathrm{~g}$. per litre (K $40 \mathrm{~m} . \mathrm{eq} / \mathrm{l} ., \mathrm{Na} 40 \mathrm{~m} . \mathrm{eq} / \mathrm{l}$, $\mathrm{Cl} .80 \mathrm{~m} . \mathrm{eq} / \mathrm{l}$ ), and (2) $\mathrm{KCl} 3.0 \mathrm{~g}$. per litre (K $40 \mathrm{~m} . e 0 / \mathrm{l}$., $\mathrm{Cl} 40$ m.eq/1.). Of these two, the first is particularly useful, and can be used with great advantage in the volume for volume replacement of observed losses, and in many cases we have given 2 litres of this solution per 24 hours with no trouble, though it is wise to control such treatment with daily serum $\mathrm{K}$ estimations until considerable experience has been obtained.

\section{The Effect of Operation}

The management of fluid and electrolyte balance in patients with intestinal obstruction is distinctly complicated if, as in many cases, an operation must be carried out. Such an operation will be followed by the usual metabolic response which follows all surgical operations. The most important features of this response may be summarized as follows: (a) For 24-48 hours there is an impaired ability to excrete water, accompanied by a diminished urine flow of raised specific gravity and electrolyte concentration; and (b) for up to 4-5 days after a major operation there is an impaired ability to excrete salt; after the initial interference with water excretion has passed off this is manifested by a markedly lowered concentration of electrolytes in the urine. As a result of these manifestations of the metabolic response to a surgical operation many of the features which 
are usuaily used as guides in the management of a fluid and electrolyte deficit temporarily lose their customary significance, especially the relationship between fluid intake and urine output, the urine specific gravity and the urine chloride concentration. Thus, usually the finding of a low urine output of high specific gravity is indicative of wäter shortage, but such findings 24 hours after release of a strangulated hernia would carry no such significance as they would most likely, under such circumstances, be no more than a manifestation of the patient's normal response to an operation. Further, if these findings are interpreted as indicating water shortage and the intake is increased, serious trouble may ensue, for, as a result of the temporarily impaired ability to excrete water, any excess water will not be excreted but will be retained in the body. Similar considerations apply with respect to the urine chloride concentration after operation.

As a result of these changes certain precautions should be taken in the post-operative period. First, during the first 24-36 hours after operation the basic intake of water should be restricted to 2 litres per 24 hours. Secondly, during this period the only reliable guides to management of fluid disturbances are the clinical condition of the patient and a careful recording of the intake and output by the various routes. Thirdly, owing to the danger of overloading, deficits of salt and water should be replaced cautiously. Fourthly, in the absence of imperative indications potassium salts should not be given intravenously during the first 36 hours after operation, owing to the low urine output during this period. Lastly, these considerations emphasize the importance, whenever possible, of replacing pre-existing deficits before operation. Quite apart from the undesirability of submitting a patient to surgery whilst still depleted of water and electrolytes, the process of replenish ment is much easier before than after operation.

\section{Conclusion}

The management of many cases of intestina obstruction will entail consideration of many factors not discussed in this brief article. Thusc in cases complicated by strangulation of bowel, i may well be necessary to give blood, plasma or $\frac{\sigma^{\circ}}{2}$ plasma substitute. Again, the actual technicap problem of administering fluids intravenously oveñ several days may require the use of special tech $\overrightarrow{0}$ niques, such as the passage of a polyethylenecatheter up into one of the great veins. Or the patient may have diabetes mellitus or somes kidney disease, which in themselves disturb the normal metabolism of water and electrolytes However, such considerations must not be allowed to distract attention from the main purw poses of treatment, namely, the administration of a rational basic intake, the assessment and replace+v ment of pre-existing losses, and the measurement and volume-for-volume replacement of observed losses. In general all this can be achieved and controlled by quite simple clinical and biochemicatw methods, of which none exceeds in importance the keeping of accurate intake and output recordo 8 well designed and accurately maintained fli ${ }^{\prime}$ balance charts form an essential background đ뮤 treatment in all cases, and in many they provie all the information that is required for successfus treatment.

\section{BIBLIOGRAPHY}

GAMBLE, J. L. (1950), 'Chemical Anatomy Physiology and Pathology of Extracellular Fluid,' Cambridge, Mass. (Harvard University Press).

LEWIS, A. A. G., SOMERVILLE, W., and LE QUESNE, L. PO (1954), Arch. Middx. Hosp., 4 , 106.

LOCKWOOD, J. S., and RANDALL, H. T. (1949), Bull. N.Y온 Acad. Med., 25, 228.

\section{RUTHIN CASTLE, NORTH WALES}

A Clinic for the diagnosis and treatment of Internal Diseases (except Mental or Infectious Diseases). The Clinic is provided with a staff of doctors, technicians and nurses.

The surroundings are beautiful. The climate is mild. There is central heating throughout. The annual rainfall is 30.5 inches, that is, less than the average for England.

The Fees are inclusive and vary according to the room occupied.

For particulars apply to THE SECRETARY, Ruthin Castle, North Wales.

Telegrams: Castie, Ruthin.

Telephone: Ruthin 66 